\title{
Possible soliton motion in ac-driven damped nonlinear lattices
}

\author{
David Cai \\ Theoretical Division and Center for Nonlinear Studies, Los Alamos National Laboratory, Los Alamos, New Mexico 87545 \\ Angel Sánchez \\ Theoretical Division and Center for Nonlinear Studies, Los Alamos National Laboratory, Los Alamos, New Mexico 87545 \\ and Escuela Politécnica Superior, Universidad Carlos III de Madrid, C./ Butarque 15, E-28911 Leganés, Madrid, Spain
}

A. R. Bishop

Theoretical Division and Center for Nonlinear Studies, Los Alamos National Laboratory, Los Alamos, New Mexico 87545

Fernando Falo and Luis M. Floría

Instituto de Ciencia de Materiales de Aragón, Consejo Superior de Investigaciones Científicas, Universidad de Zaragoza, 50009 Zaragoza, Spain

(Received 3 May 1994; revised manuscript received 8 July 1994)

\begin{abstract}
We study the possibility of kink motion induced by pure ac driving in damped nonlinear lattices, focusing on the Frenkel-Kontorova and Toda models. Numerical simulations of the Frenkel-Kontorova model show no evidence for steady kink motion. We point out that momentum-balance in addition to energy-balance arguments are needed to understand the translation of the collective excitation. Finally, our result is discussed in view of related theoretical predictions and numerical simulations.
\end{abstract}

\section{INTRODUCTION}

The relevance of nonlinear models to many problems in condensed-matter physics is nowadays widely recognized. ${ }^{1}$ One class of such models that has received considerable attention lately is that of nonlinear lattices. ${ }^{2}$. These have been mainly used in connection with commensurateincommensurate transitions, localization, and other phenomena where lattice discreteness plays an essential role. Among these models, the Frenkel-Kontorova (FK) chain $^{3}$ and the Toda lattice ${ }^{4}$ (TL) are two of the most studied ones. The motivation for these studies stems from the fact that the two models capture a number of interesting features of some condensed-matter problems, like nonlinear effects on lattice vibrations in crystals or the motion of dislocations. Most of the work to date deals with the motion of solitons and dislocations (kinks) subject to dc driving. The case of ac driving has been recently considered in Ref. 5 for the TL and in Ref. 6 for the FK chain. This last model was also studied in the presence of simultaneous ac and dc driving in Ref. 7. Restricting ourselves to pure ac driving, in the above papers a theoretical analysis of the problem with a spatially homogeneous or staggered ac driving was developed, and on the basis of these calculations it was suggested that this pure ac driving might support steady motion of solitons (TL) or kinks (FK). To our knowledge, there has been no direct numerical or experimental work to check this prediction. Therefore, we addressed the task of making a detailed check of this possibility. We note that in Ref. 9, experiments on a nonlinear electrical line related to but different from the TL showed that it was possible to drive solitons with pure ac driving. Results were also confirmed by numerical simulations. ${ }^{10}$ We will return to this point in our conclusions after we have reported our results.
This paper is organized as follows. In Sec. II, we report some simulations of the ac-driven damped FK chain, which we take as our working example. Our numerical work provided no evidence supporting the possibility of kink steady motion in this system over a wide range of parameter values. To explain this negative result, in Sec. III we develop a theoretical approach based on momentum-balance equations for both the TL and the FK chain. Our analysis shows that steady motion is indeed not possible. However, the same argument suggests that some of the results in Ref. 6 for parametric driving may be possible. Our simulations support the steady motion of kinks induced by parametric ac driving. In closing, we summarize our results and draw some more general conclusions on ac-driven nonlinear problems.

\section{MODEL AND NUMERICAL RESULTS}

For the sake of definiteness, in this section we will be dealing with the FK chain; later we will comment on the TL model. The ac-driven damped Frenkel-Kontorova chain is governed by the equation

$$
\ddot{x}_{n}=-\alpha \dot{x}_{n}-\sin x_{n}+a^{-2}\left(x_{n+1}+x_{n-1}-2 x_{n}\right)+F \cos (\omega t),
$$

where $x_{n}$ stands for the displacement of particle $n$, $n=1, \ldots, N$, and $a^{-2}$ is the stiffness of the linear interparticle interaction. Periodic boundary conditions (modulo $2 \pi$ ) are used. Equation (1) can also be realized as a discretization of the ac-driven, damped sine-Gordon (SG) equation; in that context, $a$ would be the mesh size or lattice spacing. We deal here with the regime in which there is only one dislocation in the system, meaning that there is one particle more than the number of wells. 

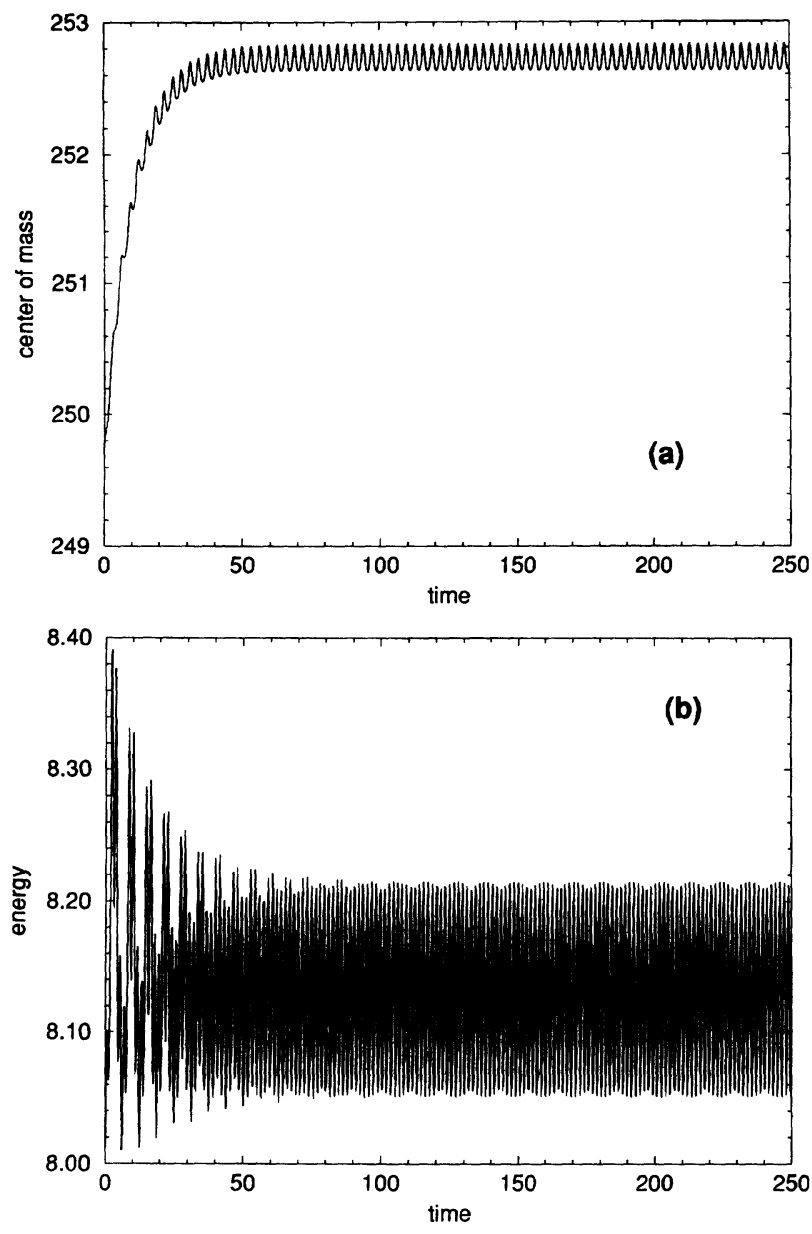

FIG. 1. (a) Center of mass motion for a kink propagating along the FK chain with initial velocity $V=0.06$. Simulation parameters are $a=0.2, F=0.1, \omega=2, \alpha=0.1$. (b) Total energy in the simulation in (a) showing its approximate balancing.

In Ref. 6 (and in Ref. 5 for the TL), this problem was addressed by means of an energy-balance calculation for a general traveling dislocation of the form ${ }^{8}$ $x_{n}(t)=\xi(2 \pi n-V t)$. It was shown that the energy-balance condition, i.e., where the dissipative losses are compensated by the ac energy input, could only be satisfied for a particular set of velocities, $V=V_{N} \equiv \omega / N$, with $N= \pm 1, \pm 2, \ldots$. It was argued that propagation of the dislocation might be possible provided the amplitude exceeds a certain threshold value $F_{N}$. The threshold was computed in the quasicontinuum limit, in which the dislocation size $\left(\sim a^{-1}\right)$ is assumed to be much larger than the chain spacing $2 \pi$. The profile $\xi$ was then taken to be the kink solution of the pure SG equation, and it yielded $F_{N}=4 \alpha \pi \tilde{V}_{N}\left(1-\tilde{V}_{N}^{2}\right)^{-1 / 2} \cosh \left[\pi \omega\left(1-\tilde{V}_{N}^{2}\right)^{1 / 2} / 2 \tilde{V}_{N}\right]$, with $\tilde{V}_{N}$ $\equiv a \omega / 2 \pi N$. It was noted that when $a \rightarrow 0$ the threshold becomes infinitely large, implying that ac-driven propagation would not be possible in the continuum limit.

Let us now turn to our results. We numerically simulated Eq. (1) by means of a fifth-order adaptive-stepsize RungeKutta routine. ${ }^{11} \mathrm{~A}$ difficult point was the choice of the parameters, which we detail in the following. First of all, the threshold is lowest when $N=1$, so we fix this value. Second, $a$ should not be very small, to keep reasonable values for the threshold, but should not be very large either, otherwise the SG kink would not be a good approximation (and it would not even be able to propagate due to the Peierls-Nabarro pinning, as discussed in Ref. 12). We chose different values in the range $0.1 \leqslant a \leqslant 1$. Finally, the resonant velocity should be proportional to $\omega$, so in order to clearly discern the kink motion, $\omega$ should be large, but not so large as to require a very small time step or give rise to velocities greater than the maximum allowed one. Thus we worked on the range $0.1 \leqslant \omega \leqslant 10$. In principle, the predictions above do not depend on $\alpha$ except for the threshold, so we usually took two typical values, $\alpha=0.01$ and 0.1 . We swept these ranges of the parameters with initial conditions given either by a kink with the expected resonant velocity, or a velocity greater than that, or at rest. All the simulations led to the same result: namely, propagation of the kink did not take place for any value of the ac-driving amplitude. An example is shown in Fig. 1; let us stress, however, that the same outcome was obtained for all the studied range of parameters, and if $a$ was taken around 1 or larger, the propagation of the kink was even more difficult due to the strong Peierls-Nabarro pinning. Actually, much before reaching the threshold $F_{N}$, the chain undergoes a transition to the chaotic regime (see, e.g., Ref. 13 for a discussion of this aspect), and the notion of a kink loses all its meaning. In this regime only large values of the dissipation can prevent the appearance of chaos. For driving amplitude values such that the kink is preserved and the input and output of the energy of the system is balanced in each period, as happens in Fig. 1(b), we always found that the kink velocity decreased (or remained zero if started at rest) and the motion eventually became oscillatory, trapped around a certain lattice point. Therefore, our numerical results strongly suggest that steady kink motion does not occur in the ac-driven damped FK chain.

\section{ANALYTICAL RESULTS AND DISCUSSION}

Bearing the above results in mind, we now turn to an analytical approach to gain further insight into this problem. Notice that the total momentum of the particles

$$
p=\sum_{n} \dot{x}_{n},
$$

the dot meaning derivative with respect to time, is directly related to the velocity of a steady traveling dislocation $x_{n}(\eta) \equiv \xi(a n-V t)$ by

$$
p=V \sum_{n}\left(-\frac{d \xi}{d \eta}\right)
$$

Therefore, this is a very good indicator of the possibility of steady motion of collective excitations. We note that this Ansatz amounts to neglecting higher order effects such as radiation and, on the other hand, it is the same one used by Bonilla and Malomed. ${ }^{6}$ It can be easily seen from Eq. (2) that 


$$
\begin{aligned}
\dot{p} & =\sum_{n} \ddot{x}_{n} \\
& =-\alpha p+\sum_{n}\left[F \cos (\omega t)-\sin x_{n}\right] .
\end{aligned}
$$

The condition for stable translation of the profile $x_{n}$ requires $\langle\dot{p}\rangle=0$, where $\langle\cdots\rangle$ means average over a period of the driving frequency. Imposing this condition on Eq. (4) leads to the following relationship:

$$
\alpha\langle p\rangle=-\left\langle\sum_{n=-\infty}^{+\infty} \sin x_{n}\right\rangle,
$$

which must hold if the considered excitation is to move with a constant velocity. Equation (5) has to be particularized for each specific profile considered. Following Ref. 6, we concern ourselves with a single dislocation motion. In the quasicontinuum approximation, the unperturbed dislocation is well described by the kink solution of the sine-Gordon (SG) equation, given by

$$
x(\eta)=4 \tan ^{-1}\{\exp [\sigma \gamma(a n-V t)]\},
$$

where $\gamma=\left(1-V^{2}\right)^{-1 / 2}$ and $\sigma$ is the polarity of the kink. By substituting Eq. (6) for $x_{n}$ into Eq. (5), it leads to

$$
\begin{aligned}
\alpha\langle p\rangle= & -\left\langle\int_{-\infty}^{+\infty} \frac{d \eta}{a} \sin [x(\eta)]\right. \\
& \left.\times\left\{1+2 \sum_{s=1}^{+\infty} \cos \left[\frac{2 \pi s}{a}(\eta+V t)\right]\right\}\right)
\end{aligned}
$$

The terms in the right-hand side of Eq. (7) can be regarded as the time average of the Fourier modes of the Peierls-Nabarro pinnings. For the kink profile (6) and the resonant velocity, $V=a \omega /(2 \pi)$ (from the energy-balance argument), the time averaged Peierls-Nabarro pinnings vanish. Therefore, the only value of $V$ which will balance the total momentum is $V=0$, and constant kink motion will not be possible. As a matter of fact, this conclusion holds for any profile $x_{n}(\eta)$ which is an odd function of $\eta$ under the quasicontinuum approximation. We also point out that the same calculation can be carried out for a periodically varying velocity, and the conclusion $(V=0)$ remains true, now in the sense of average velocity.

Notice that the contradiction with the results in Ref. 6 arises because the way the resonant velocities are obtained there is by imposing energy balance, not momentum balance. The fact that there could be Ansätze with constant speed that could balance the energy in the system is not enough to guarantee that they are true solutions of the system. Indeed, in Ref. 6 another example is considered for a continuum model, described by a perturbed SG equation with a parametric driving of the form $F \sin (\phi / 3) \cos (\omega t)$ ( $\phi$ being the SG field) by means of a similar momentum-balance approach. It is predicted that steady motion of kinks can take place. We have checked this possibility by direct numerical simulation, and, opposite to the previous case, the result agrees with the prediction in Ref. 6. In spite of the fact that the kink velocity is not constant, it certainly exhibits motion towards a preferred direction.

Turning now to the TL, its governing equation in the presence of ac driving and damping can be written as

$\ddot{y}_{n}=-\alpha \dot{y}_{n}-e^{-\left(y_{n+1}-y_{n}\right)}+e^{-\left(y_{n}-y_{n-1}\right)}+F_{n} \cos (\omega t)$,

where $F_{n}=F$ for a spatially uniform drive, or $F_{n}=(-1)^{n} F$, a staggered drive as done in Refs. 5, 9, and 10. We can now apply the same momentum-balance approach to the perturbed TL model. The momentum $p=\Sigma \dot{y}_{n}$ satisfies

$$
\dot{p}=-\alpha p
$$

for the staggered force. For the spatially uniform drive, the above equation also holds in the time averaged sense. For a pure Toda lattice soliton,

$$
y_{n}=-\ln \left[\frac{1+e^{-2 a} e^{-2 a\left(n-V t-x_{0}\right)}}{1+e^{-2 a\left(n-V t-x_{0}\right)}}\right],
$$

where $V=\epsilon \sinh a / a, a>0, \epsilon= \pm 1$, the magnitude of the momentum is

$$
|p|=2 a|V|=2 \sinh a .
$$

From Eq. (9), it follows that the decay of the magnitude of the momentum leads to the decay of $a$, thereby, the decay of $V$, that is, a broadening of the soliton with a decreasing velocity in time. Thus the ac drive again cannot sustain the velocity of the TL soliton.

\section{CONCLUSION}

In this paper, we have shown that according to the momentum-balance approach, it is not possible to sustain kinks (solitons) in the FK chain (TL) by means of pure ac driving. Similar results hold for the purely ac-driven, damped nonlinear Schrödinger and Ablowitz-Ladik systems. ${ }^{14}$ Numerically, in the studied range of parameters for the FK chain, we have never observed such a steady motion, but rather oscillatory motion around some lattice point which is the initial one if starting from $V=0$ or a different one, which is reached after the decay of the initially nonzero velocity. We believe that earlier suggestions that this kind of phenomenon should occur are not correct as they use only an energy-balance instead of a momentum-balance condition. We have also verified numerically some of these earlier predictions that were obtained by this momentumbalance approach.

From a more general viewpoint, it seems that the propagation of collective excitations in nonlinear lattices with pure ac driving is unlikely. Only special lattices, or parametric drivings which somehow break the symmetry of the excitation making it possible to choose a preferred direction, would be good candidates for exhibiting this phenomenon. In this respect, it is worth mentioning the work in Refs. 9 and 10, where experiments on a nonlinear electrical lattice and numerical simulations were conducted, showing that solitons could propagate by using pure ac driving. The system actually simulated is dual to the lattice which is obtained from the TL by a change of variables, $r_{n} \equiv y_{n}-y_{n-1}$ [see Eq. (8)], 
such that it admits all TL solutions as a subset. The equation of motion now is

$$
\ddot{r}_{n}=-\alpha \dot{r}_{n}-e^{-r_{n+1}}-e^{-r_{n-1}}+2 e^{-r_{n}+2(-1)^{n} F} \cos (\omega t) .
$$

Once again for a steady translating profile $r_{n}(\eta)$ $=r(n-V t)$, the momentum $p=\Sigma \dot{r}_{n}=V \Sigma\left(-d r_{n} / d \eta\right)$, which is conserved for the unperturbed system, satisfies

$$
\dot{p}=-\alpha p
$$

but

$$
p \equiv 0
$$

for a one-soliton solution, $r_{n}=-\ln \left[1+\Omega^{2} \operatorname{sech}^{2}(k \eta)\right]$, where $\Omega^{2}=\sinh ^{2} k, V=\Omega / k$. Actually, $p \equiv 0$ for any solutions of the original TL equation. Hence Eq. (13) no longer imposes any constraint on the evolution of the soliton parameters. This admits the possibility of a steady translating soliton driven by the staggered force, as long as the drive can supply suf- ficient energy to maintain the amplitude of the soliton against the damping. As we can see, the possibility of an ac-driven constant velocity cannot be excluded in general, although we believe it should be regarded as an exceptional case.

\section{ACKNOWLEDGMENTS}

We are indebted to L. L. Bonilla who got us interested in this problem, and to N. Gronbech-Jensen, B. A. Malomed, and R. D. Parmentier for fruitful discussions. A.S. acknowledges the Universidad de Zaragoza for warm hospitality where part of this work was done, and partial support from a MEC/Fulbright grant, from DGICyT (Spain) under Project No. PB92-0248, and from the European Union Network ERBCHRXCT930413. Work at Zaragoza was supported by DGICyT (Spain) under Project No. PB92-0361, and by the European Union (NETWORK on Nonlinear Approaches to Coherent and Fluctuating Processes in Condensed Matter and Optical Physics). Work at Los Alamos was performed under the auspices of the U.S. Department of Energy.
${ }^{1}$ Microscopic Aspects of Nonlinearity in Condensed Matter, edited by A. R. Bishop, V. L. Pokrovsky, and V. Tognetti (Plenum Press, New York, 1991); Nonlinearity in Materials Science, edited by A. R. Bishop, R. Ecke, and J. Gubernatis (NorthHolland, Amsterdam, 1992).

${ }^{2}$ P. Bak, Rep. Prog. Phys. 45, 587 (1982); W. Selke, Phys. Rep. 170, 213 (1988); W. Selke, in Phase Transitions and Critical Phenomena, edited by C. Domb and J. L. Lebowitz (Academic, New York, 1993).

${ }^{3}$ Ya. I. Frenkel and T. Kontorova, Zh. Eksp. Teor. Fiz. 8, 1340 (1938); F. C. Frank and J. H. van der Merwe, Proc. R. Soc. London Ser. A 189, 205 (1949).

${ }^{4}$ M. Toda, J. Phys. Soc. Jpn. 22, 431 (1967); 22, 501 (1967); Phys. Rep. 18, 1 (1973).

${ }^{5}$ B. A. Malomed, in Microscopic Aspects of Nonlinearity in Condensed Matter, edited by A. R. Bishop, V. L. Pokrovsky, and V. Tognetti (Plenum Press, New York, 1991); Phys. Rev. B 45,
4097 (1992).

${ }^{6}$ L. L. Bonilla and B. A. Malomed, Phys. Rev. B 43, 11539 (1991).

${ }^{7}$ L. M. Floría and F. Falo, Phys. Rev. Lett. 68, 2713 (1992).

${ }^{8}$ The notation of Ref. 6 is used in this paragraph for the convenience of comparison.

${ }^{9}$ T. Kuusela, Phys. Lett. A 167, 54 (1992).

${ }^{10}$ T. Kuusela, J. Hietarinta, and B. A. Malomed, J. Phys. A 25, L21 (1993).

${ }^{11}$ W. H. Press, S. A. Teukolsky, W. T. Vetterling, and B. P. Flannery, Numerical Recipes in C, Second Edition (Cambridge University Press, New York, 1992).

${ }^{12}$ M. Peyrard and M. D. Kruskal, Physica D 14, 88 (1984).

${ }^{13}$ J. C. Ariyasu and A. R. Bishop, Phys. Rev. B 35, 3207 (1987); Phys. Rev. A 39, 6409 (1989).

${ }^{14}$ D. Cai, A. R. Bishop, N. Grønbech-Jensen, and B. A. Malomed, Phys. Rev. E 50, 694 (1994). 

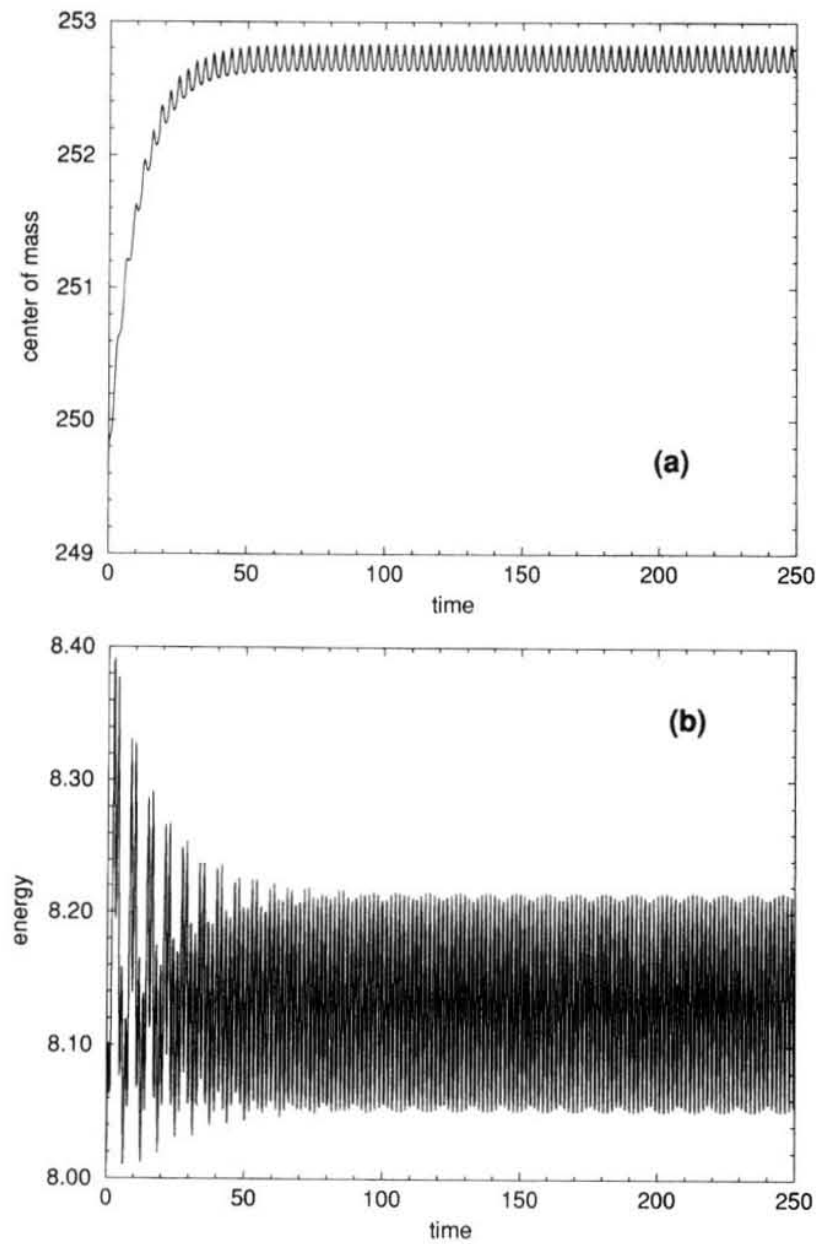

FIG. 1. (a) Center of mass motion for a kink propagating along the FK chain with initial velocity $V=0.06$. Simulation parameters are $a=0.2, F=0.1, \omega=2, \alpha=0.1$. (b) Total energy in the simulation in (a) showing its approximate balancing. 